Xia et al.

\title{
1 Enhanced MATE transporter DTX6/PQT15 confers paraquat
}

2 resistance

3 Jin-Qiu Xia, Tahmina Nazish, Ayesha Javaid, Mohsin Ali, Qian-Qian Liu, Liang Wang,

4 Zheng-Yi Zhang, Zi-Sheng Zhang, Yi-Jie Huang, Jie Wu, Zhi-Sen Yang, Lin-Feng

5 Sun, Yu-Xing Chen, Cheng-Bin Xiang

6

7 School of Life Sciences and Division of Molecular \& Cell Biophysics, Hefei National

8 Science Center for Physical Sciences at the Microscale, University of Science and

9 Technology of China, The Innovation Academy of Seed Design, Chinese Academy of

10 Sciences, Hefei, Anhui Province 230027, China

11

12 Corresponding author:

13 Cheng-Bin Xiang (Email: xiangcb@ ustc.edu.cn; Phone: 86-551636-00429)

14

15 
Xia et al.

\section{ABSTRACT}

Paraquat is one of the most widely used non-selective herbicides and has elicited the emergence of paraquat resistant weeds. However, the molecular mechanisms of paraquat resistance are not completely understood. Here we report an Arabidopsis gain-of-function mutant pqt15-D with significantly enhanced resistance to paraquat and the corresponding PQT15 encoding the MATE transporter DTX6. A point mutation at +932 bp in DTX6 causing the G311E amino acid residue change brings about the enhanced paraquat resistance of pqt15-D. Overexpression of DTX6/PQT15

in the wild type also confers strong paraquat resistance, whereas the DTX6/PQT15 knockout mutants exhibits hypersensitive phenotype to paraquat. Moreover, heterologous expression of DTX6 and DTX6-D in E. coli significantly enhances bacterial resistance to paraquat. DTX6/PQT15 mainly localizes in the plasma membrane as shown by DTX6-GFP and functions as a paraquat efflux transporter as demonstrated by paraquat efflux assays with isolated protoplasts and bacterial cells. Taken together, our results demonstrate that DTX6/PQT15 is an efflux transporter and confers paraquat resistance by exporting paraquat out of cytosol, therefore unraveling a molecular mechanism of paraquat resistance in higher plants and providing a promising candidate of generating paraquat resistance crops.

Key words: paraquat, paraquat resistance, DTX/MATE transporter subfamily, AtDTX6/PQT15 


\section{INTRODUCTION}

Paraquat (or methyl viologen; 1,1'-dimethyl-4,4' bipyridinium dichloride), a strong inducer of oxidative stress, is a widely used broad spectrum, quick-acting, nonselective herbicide. It quickly competes for electrons from PS I (photosynthesis system I) and reacts with oxygen, producing large quantity of reactive oxygen species (ROS) that destroy the photosynthesis system and ultimately kill plants (Hawkes, 2014; Suntres., 2002).

Paraquat resistance has been reported in weeds and Arabidopsis (Chen et al., 2009; Fujita et al., 2012; Itoh and Miyahara, 1984; Li et al., 2013; Luo et al., 2016; Murgia et al., 2004; Xi et al., 2012). Based on physiological studies of paraquat resistant weeds, several resistance mechanisms have been proposed, including translocation, sequestration, catabolism, and ROS scavenging (Fujita and Shinozaki, 2014; Hawkes, 2014; Murgia et al., 2004; Szigeti and Lehoczki, 2003). Although no genetic loci of paraquat resistance have been identified in weeds, some of the proposed mechanisms have been confirmed in Arabidopsis. For instance, AtPDR11 encodes a plasma membrane-localized ATP-binding cassette transporter that uptakes paraquat from environment into cells. Therefore, knockout of AtPDR11 confers improved paraquat resistance (Xi et al., 2012). Some L-type amino acid transporters also transport paraquat and confer paraquat resistance when their function is lost (Fujita et al., 2012; Li et al., 2013). Enhanced capability of ROS scavenging was also confirmed by Arabidopsis mutants (Chen et al., 2009; Luo et al., 2016). So far, other proposed mechanisms have not been confirmed.

The Detoxification Efflux Carriers (DTX)/Multidrug and Toxic Extrusion (MATE) subfamily genes are universally present in plants, animals and microorganisms and perform a variety of functions (Omote et al., 2006; Yuji Morita et al., 2000). There are more than 50 DTX members in the Arabidopsis genome (Li et al., 2002). The DTX subfamily proteins contain 3 to 12 signature transmembrane domains (Lu, 2016; Lu et al., 2018; Miyauchi et al., 2017; Tanaka et al., 2013), localize to various cell membranes or organelles (Dobritzsch et al., 2016; Li et al., 2002; 
Marinova et al., 2007; Serrano et al., 2013; Zhang et al., 2017; Zhang et al., 2014), and transport different sorts of substrates (Dobritzsch et al., 2016; Magalhaes et al., 2007; Roschzttardtz et al., 2011; Serrano et al., 2013). For example, AtDTX1 in plasma membrane participates in the efflux of plant-derived alkaloids, antibiotics and other toxic compounds in Arabidopsis (Li et al., 2002). DTX50 facilitates ABA efflux and thus modulates ABA sensitivity and drought tolerance (Zhang et al., 2014). Recently, rice DG1, a MATE transporter too, was reported to play a key role in grain filling by mediating ABA long distance transport from leaf to caryopsis (Qin et al., 2021). DTX33 and DTX35 function as chloride channels and regulate cell turgor in Arabidopsis (Zhang et al., 2017). AtFRD3 and OsFRDL1 mediate citrate efflux and thus the response to iron deficiency (Durrett et al., 2007; Green and Rogers, 2004; Rogers and Guerinot, 2002; Yokosho et al., 2009). In addition, some DTXs have been shown to mediate various toxins resistance ( $\mathrm{Li}$ et al., 2002; Omote et al., 2006). For instance, AtALF5 confers resistance to tetramethylammonium in yeast (Diener. et al., 2001). AtDTX1 enhances the tolerance to norfloxacin and heavy metal cadmium in $E$. coli KAM3 strain (Li et al., 2002). Some DTXs/MATEs can positively regulate aluminum tolerance by enhancing citrate secretion (Doshi et al., 2017; Li et al., 2018; Li et al., 2017; Ma et al., 2018; Yokosho et al., 2011).

Here, we report the isolation and characterization of the gain-of-function mutant paraquat tolerance 15-D (pqt15-D) with enhanced resistance to paraquat. PQT15 encodes AtDTX6 protein in the DTX/MATE family of Arabidopsis. A point mutation in PQT15 results in G311E amino acid residue change, leading to paraquat resistance in pqt15-D. We further showed that overexpression of DTX6/PQT15 also confered paraquat resistance in the wild type, whereas the DTX6/PQT15 knockout mutants exhibited hypersensitive phenotype to paraquat. Heterologous expression of DTX6 and DTX6-D in E. coli also significantly enhanced bacterial resistance to paraquat. Paraquat efflux assays with isolated protoplasts and bacterial cells directly demonstrated the paraquat efflux activity of DTX6/PQT15. Taken together, our results demonstrate that DTX6 acts as a efflux transporter and confers paraquat resistance by 
exporting paraquat out of cytosol, therefore revealing a molecular mechanism of paraquat resistance that has not been reported before in higher plants.

\section{RESULTS}

\section{Isolation and characterization of pqt15-D with enhanced paraquat resistance}

To obtain more paraquat resistance loci, we carried out a near saturation mutagenesis with EMS (ethyl methyl sulfonate) in the Columbia ecotype wild type background (125,000 seeds mutagenized). Using our previously established method (Xi et al., 2012), we screened the whole M2 mutant library under $2 \mu \mathrm{M}$ paraquat selection pressure and isolated dozens of paraquat resistant mutants. To identify novel loci of paraquat resistance, we performed genomic DNA sequencing of those known paraquat resistant loci for each mutant, including PARl (Li et al., 2013), RMVI (Fujita et al., 2012), AtPDR11 (Xi et al., 2012), and PQT3 (Luo et al., 2016). To our surprise, the whole genetic screen ended up with only 2 major paraquat resistant loci. One is parl with more than 20 independent mutants each with a distinct mutation. The other is a novel locus represented by pqt15-5 with 8 independent mutants but all with the same point mutation (Table S1).

We re-screened the progeny of the pqt15-5 and confirm the paraquat resistance phenotype (Figure 1a). Then the mutant was backcrossed with Col-0 wild type to get $\mathrm{BC} 1 \mathrm{~F} 1$ generation, and $\mathrm{F} 1$ plants were selfed to get $\mathrm{BC} 1 \mathrm{~F} 2$ generation. $\mathrm{BC} 1 \mathrm{~F} 2$ population was phenotyped for paraquat resistance on MS medium containing $2 \mu \mathrm{M}$ paraquat (Figure 1b). The segregation ratio of resistance: sensitive $\approx 3: 1$ (survived: dead $\left.=230: 88 ; \chi^{2}=1.07<\chi_{(0.05)}^{2}=3.84\right)$. The genetic analysis showed that the pqt15-5 is caused by a single dominant nuclear gene mutation. Therefore, we renamed the mutant pqt15-D.

To identify the mutation associated with paraquat resistance in the pqt15-D mutant, we pooled 50 paraquat resistant plants in BC1F2 population (more than 300 seeds) for whole-genome sequencing and identified a G-A substitution at position 
+932 bp in the coding region of DTX6, resulting in G311E replacement in amino acid thus further confirming that this point mutation caused the paraquat resistance phenotype in pqt15-D.

\section{DTX6/PQT15 is primarily expressed in roots and DTX6/PQT15 is mainly} localized in plasma membrane

To examine the expression pattern of DTX6/PQT15, its transcript levels were detected for various plant tissues including roots, stem, rosette leaves, cauline leaves, flower, siliques, and seeds by quantitative RT-PCR analyses. The result shown that $D T X 6 / P Q T 15$ was primarily expressed in the root and the expression levels were low in other tissues (Figure 2a).

To analyze the subcellular localization of DTX6/PQT15, we generated DTX6pro:DTX6-GFP transgenic plants and found GFP signals in the plasma membrane and inner membranes of root cells (Figure 2b, c), indicating that DTX6-GFP fusion protein is localized in the plasma membrane and inner membranes in the root.

DTX6/PQT15 confers paraquat resistance in Arabidopsis and E. coli

To verify genetically the function of DTX6, we obtained two knockout mutant lines ( $d t x 6-1$ and $d t x 6-2)$ with CRISPR technology and three $35 S$-DTX6 transgenic Arabidopsis lines (OE-9, OE-13 and OE-17) in wild type background (Figure S2). germination assay. There was no significant difference of survival among the lines when germinated on MS medium without paraquat. However, when germinated on MS medium containing paraquat of different concentrations (1.0, 1.5, and $2.0 \mu \mathrm{M})$, $d t x 6-1$ and $d t x 6-2$ lines were more sensitive to paraquat, whereas the OE-9, OE-13 and OE-17 showed significantly enhanced resistance to paraquat compared with wild type. Meanwhile, pqt15-D showed similar phenotype with OE lines (Figure 3a and b). 
dtx6-1, dtx6-2, wild type (Col-0), pqt15-D, and OE lines (OE-9, OE-13, and OE-17) wild type showed sensitive phenotype to paraquat, whereas the OE lines displayed significantly enhanced resistance with $100 \%$ vs. $<5 \%$ survival of wild type (Figure 3c bottom and d), but pqt15-D only showed slightly improved resistance to paraquat with $20 \%$ survival compared with wild type (Figure $3 \mathrm{c}$ and d) apparently due to the low expression level of DTX6 in the leaf (Figure S3a \& b, Figure 2a).

Moreover, E. coli expressing DTX6 also displayed enhanced resistance to paraquat $(200 \mu \mathrm{M})$ while DTX6-D confered even higher resistance (Figure 3e), further demonstrating the effect of the point mutation on paraquat resistance.

\section{DTX6/PQT15 effluxes paraquat out of the cell}

Given that DTX6/PQT15 is a member of DTX/MATE subfamily transporters, which are known to participate in organic compounds and xenobiotic toxin efflux, we speculated that DTX6/PQT15 might function as a paraquat efflux transporter. To confirm the hypothesis, we conducted efflux assay with mesophyll protoplasts prepared from wild type (Col-0) and $\mathrm{OE}-13$ plant leaves. Radiolabeled ${ }^{14} \mathrm{C}$-paraquat was preloaded into mesophyll protoplasts, and then ${ }^{14} \mathrm{C}$-paraquat was measured by monitoring the radioactivity retained in the protoplasts and released into the medium. The results showed that the rate of ${ }^{14} \mathrm{C}$-paraquat retention was significantly lower in

175 OE-13 protoplasts (Figure 4a), while rate of ${ }^{14} \mathrm{C}$-paraquat release from OE-13

176 protoplasts was significantly faster than that from the wild type (Figure 4b). expressing DTX6 or DTX6-D. Both DTX6 and DTX6-D were able to export paraquat out of the bacterial cells (Figure 4c).

To understand the mechanism of DTX6-D enhanced paraquat transport, we 
resolved crystal structure of AtDTX14 (Miyauchi et al., 2017), which shares a sequence identity of $50.9 \%$ with DTX6, and thus is considered as a suitable model for DTX6 and DTX6-D (Figure 5a). In the model, the residue Gly311 is localized in the entrance of the substrate tunnel. However, the variant G311E in DTX6-D would increase negative charge of the entrance (Figure 5b-d). Therefore, we hypothesize that the increased negative charge could favor the recognition of the positively charged paraquat and eventually increase the transport activity of DTX6. To prove this speculation, we mutated the Gly at 311 position to negatively charged glutamic acid, aspartic acid, and uncharged glutamine, respectively, then heterologously expressed in E. coli. Compared with the cells transformed with pET28a (empty vector), replacement with negatively charged glutamic acid or aspartic acid enhanced paraquat resistance of $E$. coli, whereas replacement with the uncharged glutamine did not enhance the resistance to paraquat compared with the wild type DTX6 on the medium with $200 \mu \mathrm{M}$ paraquat (Figure 5e, f).

\section{DISSCUSSION}

In this study, we report the isolation and characterization of a gain-of-function mutant pqt15-D (or $d t x 6-D$ ) with enhanced paraquat resistance. Using the MutMap method (Takagi et al., 2015), we identified a G to A point mutation at position +932 in the coding region of $D T X 6 / P Q T 15$ encoding a MATE transporter, which caused the enhanced paraquat resistance phenotype in pqt15-D (Figure 1, Figure S1). Moreover, the enhanced paraquat resistance can also be achieved by overexpressing DTX6 in Arabidopsis and E. coli (Figure 3). We further revealed the molecular mechanism underlying the DTX6/PQT15-conferred paraquat resistance by paraquat efflux assays with mesophyll protoplasts and E. coli cells, which clearly showed that DTX6 and DTX6-D were able to export paraquat out of the cell (Figure 4), reducing cellular paraquat concentration and thus alleviating its toxic effects. Consistent with this, DTX6-GFP fusion protein was mainly localized to the plasma membrane (Figure 2).

Therefore, our results have unraveled a molecular mechanism of paraquat resistance 
in higher plants that has not been reported before.

214 Considering DTX6 as a member of MATE transporter subfamily, which has been 215 reported to transport kinds of organic compounds in bacteria, plants and animals

216 (Kuroda and Tsuchiya, 2009; Li et al., 2002; Omote et al., 2006), DTX6 may be 217 capable of effluxing paraquat from the cytosol to the extracellular space. Indeed, as 218 shown by the efflux assays, the wild type DTX6 is able to export paraquat out of the 219 protoplasts (Figure 2a, b), but the paraquat efflux activity is likely too low to confer paraquat resistance in wild type. Once its activity is enhanced either by the point mutation as in the pqt15-D mutant or elevated expression as in the overexpression

222 lines, strong paraquat resistance can be conferred (Figure 3). What are the natural substrates of DTX6 is an interesting question to pursue. It is conceivable that its natural substrates are quite likely positively charged compounds.

The subcellular localization of DTX6 is in agreement with its role as an efflux transporter. Our DTX6pro:DTX6-GFP transgenic plants clearly show that TDX6-GFP

227

228

229

230

231

232

233

234

235

236

237

238

239

240

241 fusion protein is mainly in the plasma membrane in the root. However, GFP signals also appeared in the inner membranes, indicating the possibility of other subcellular location of DTX6. Since the DTX6 promoter activity is very low in the leaf, we could not detect GFP signals in the leaf with our DTX6pro:DTX6-GFP transgenic plants. Considering the strong paraquat resistance of the OE lines, it is conceivable that DTX6 would similarly localize in plasma membrane in the leaf but would be interesting to see where else DTX6-GFP is subcellularly localized.

How could a single amino acid change (G311E) of DTX6 afford Arabidopsis resistant to paraquat? Since the G311E single amino acid replacement predicted in the substrate entry domain is solely responsible for enhanced paraquat resistance in the mutant, we reasoned that electrical charge from neutral to negative would favor the binding of DTX6 to paraquat, thus increase DTX6 protein efflux activity and confer higher resistance to paraquat (Figure 3e). Indeed, G311D substitution in DTX6 could also confer enhanced paraquat resistance (Figure 5f), suggesting that a more negatively charged surface at the substrate entry would favor paraquat interaction with 
DTX6, which may enhance efflux activity of DTX6.

In summary, with the pqt15-D mutant, we unraveled DTX6 as a paraquat efflux

transporter. No paraquat efflux transporters of higher plants have been reported before.

Paraquat efflux out of the cell represents a new mechanism for paraquat resistance in higher plants. We have demonstrated that enhanced DTX6/PQT15 via either the

G311E amino acid alteration as in pqt15-D or elevated expression of DTX6/PQT15 as

in the $\mathrm{OE}$ lines would confer strong resistance to paraquat in Arabidopsis and E. coli.

Undoubtedly, this will provide a promising candidate gene for engineering paraquat resistance crops.

\section{MATERIALS AND METHODS}

\section{Plant materials and growth conditions}

Arabidopsis Columbia ecotype (Col-0) was used in this study. Two mutants $d t x 6-1$ and $d t x 6-2$ were obtained via CRISPR/Cas9 technology (Figure S2a-c). The transgenic plants were obtained by transferring DTX6pro:DTX6-GFP and 35S-DTX6 constructs into Arabidopsis using the floral dip method (Bent., 2000). Plants were grown in soil under greenhouse condition $\left(22^{\circ} \mathrm{C} ; 16 / 8 \mathrm{~h}\right.$ light/dark cycle for long-day; 8/16 h light/dark for short-day; with light intensity $100 \mu \mathrm{M} \mathrm{m}^{-2} \mathrm{~s}^{-1}$ ) or MS medium containing $0.5 \%$ agar and $1 \%$ sucrose in the growth chamber $\left(22{ }^{\circ} \mathrm{C} ; 16 / 8 \mathrm{~h} \mathrm{light/dark}\right.$ cycle for long-day; $100 \mu \mathrm{M} \mathrm{m}^{-2} \mathrm{~s}^{-1}$ light intensity).

Genetic screen and identification of mutation associated with paraquat resistance in the mutants

More than 1,000,000 EMS-mutagenized M2 seeds were germinated and grown for 7-10 days on MS medium containing $2 \mu \mathrm{M}$ paraquat to screen for paraquat resistant mutants. In the germination phase, the vast majority of seedlings are bleached or cannot product roots, whereas candidate paraquat resistant mutants stay green and produce roots. The candidate paraquat resistant mutants were transferred to soil for M3 seeds. M3 seeds were rescreened under same condition, and then were 
used to backcross with wild type for F1 seeds and F2 was further obtained through F1

272 selfing.

273 The F2 population was phenotyped by using the same screening condition and 50 resistant plants were pooled to extract genomic DNA for whole genome re-sequencing by the BSA method (Abe et al., 2012; Takagi et al., 2015). Genome re-sequencing was commercially done by Weifen (Anhui) Gene Technology Co. (Hefei, China).

Paraquat resistance assay of seed germination

For paraquat resistance assay of seeds germination, seeds were surface-sterilized for $10-15 \mathrm{~min}$ in $10 \%$ bleach, washed five times with sterile water, and seeds were

280

281

282

283

284

285

286

287

288

289

290

291

292

293

294

295

296

297

298

299

vernalized at $4^{\circ} \mathrm{C}$ for $48 \mathrm{~h}$. After vernalization, seeds were plated on MS solid medium with $1 \%(\mathrm{w} / \mathrm{v})$ sucrose, $0.5 \%(\mathrm{w} / \mathrm{v})$ agar or MS medium containing different concentration paraquat $(1.0,1.5$ and $2.0 \mu \mathrm{M})$. After one week, the survived was counted. Survival was defined as percent of seedlings with green cotyledon.

\section{Paraquat spray assay of soil grown plants}

4-weeks-old plants grown in soil were sprayed with $80 \mu \mathrm{M}$ paraquat solution.

After 5 days, the survived was counted. Survival was defined as percent of plants with green leaves.

\section{Quantitative RT-PCR analyses}

Total RNA was extracted from different tissues of plants with Trizol method (Invitrogen, Carlsbad, USA). After that total RNA is reversely transcribed into cDNA by TransScript Kit (TaKaRa, Dalian, China). The specific primers were used for RT/qRT-PCR (Table S1).

\section{Subcellular localization of DTX6-GFP fusion protein}

Roots of DTX6pro:DTX6-GFP transgenic plants were observed by using ZEISS880 confocal laser scanning microscope as described (Chaiwanon et al., 2016; Zhao et al., 2020). Protoplasts were prepared from the transgenic roots as described (Yoo et al., 2007) and observed as above.

\section{${ }^{14} \mathrm{C}$-paraquat efflux assay in Arabidopsis protoplasts}

Arabidopsis mesophyll protoplasts were isolated from Arabidopsis rosette leaves 
of Col-0 and OE-13 lines grown for 4 weeks in a short day photoperiod as described (Yoo et al., 2007). About $6 \times 10^{6}$ of mesophyll protoplasts were incubated for $1 \mathrm{~h}$ in 2 $\mathrm{ml}$ bathing W5 solution (154 mM NaCl, $125 \mathrm{mM} \mathrm{CaCl}_{2}, 5 \mathrm{mM} \mathrm{KCl}$ and $2 \mathrm{mM} \mathrm{MES}$, pH 5.7) with $3 \mu \mathrm{M}{ }^{14} \mathrm{C}$-paraquat (American Radiolabeled Chemicals, Inc., Saint Louis, USA) at room temperature. Then, the mesophyll protoplasts were washed three times with ice cold bath W5 solution to remove unloaded ${ }^{14} \mathrm{C}$-paraquat and resuspended in $13 \mathrm{ml}$ bath W5 solution with protoplast concentration of $5 \times 10^{5}$ per $\mathrm{ml} .{ }^{14} \mathrm{C}$-paraquat release from the protoplasts was monitored by removing $1 \mathrm{ml}$ aliquot sample in triplicate at different time points $(0,10,20$ and $40 \mathrm{~min})$ at room temperature. Immediately, the aliquoted samples were pulse spinned to separate protoplasts from the bath solution, then the protoplast pellet and supernatant were separately mixed with $5 \mathrm{ml}$ scintillation solution to measure radioactivity by liquid scintillation counter (Perkin-Elmer Tri-carb 2910TR, Waltham, USA).

\section{Paraquat resistance and ${ }^{14} \mathrm{C}$-paraquat efflux assays of $E$. coli ROSETTA strain} expressing DTX6 and DTX6-D

The DTX6, DTX6-D, DTX6-Asp, and DTX6-Gln cDNA were constructed into the pET28a vector, respectively, and introduced into the $E$. coli ROSETTA strain and grown on solid LB medium with $50 \mathrm{mg} / \mathrm{ml}$ kanamycin antibiotics, single clones were picked, incubated in liquid medium containing $50 \mathrm{mg} / \mathrm{ml}$ kanamycin and $1 \mathrm{mM}$ IPTG to $\mathrm{OD}_{600}=0.4$, then $2 \mu 1$ of the 1:10 diluted culture was spotted on LB solid medium containing $50 \mathrm{mg} / \mathrm{ml}$ kanamycin, $1 \mathrm{mg} / \mathrm{ml} \mathrm{IPTG}$, and different concentrations of paraquat and incubated for $24 \mathrm{~h}$ at $37^{\circ} \mathrm{C}$ before colony growth was checked. The ROSETTA strain with pET28a empty plasmid was used as a control.

$\mathrm{A}{ }^{14} \mathrm{C}$-paraquat transport assay was performed as described previously (Zhang et al., 2014). DTX6 and DTX6-D were expressed in E. coli ROSETTA strain, respectively, and then $5 \mu \mathrm{M}{ }^{14} \mathrm{C}$-paraquat was loaded into the ROSETTA strains with pET28a (control), pET28a-DTX6, and pET28a-DTX6-D plasmid, respectively.

Paraquat release from bacterial cells was monitored by removing aliquot samples at different time points $(0,10,30$ and $60 \mathrm{~min})$ at room temperature. After that, the 
sample was pulse spinned, then the supernatant was mixed with $5 \mathrm{ml}$ scintillation Tri-carb 2910TR, Waltham, USA).

332

333

\section{D-molecular models of DTX6 and DTX6-D}

Using AtDTX6 homologous proteins AtDTX14 as the most suitable molecular structural templates (Protein Data Bank accession 5Y50). AtDTX14 has norfloxacin export activity like AtDTX1 (Li et al., 2002; Miyauchi et al., 2017). 3D-molecular models of DTX6 and DTX6-D were constructed with Phyre2 software.

\section{Statistical analysis}

Statistical significance was evaluated at the 0.05 probability level using Student's t-test.

\section{AUTHOR'S CONTRIBUTION}

JQX and CBX designed the experiments. JQX performed experiments and data analysis, and wrote the manuscript. TN, AJ, MA, QQL, ZYZ, ZSZ and JW contributed to assist in completing part of the experiment. LW, YXC, ZSY, and LFS contributed to construct 3D-molecular models. CBX supervised the project and revised the manuscript.

\section{ACKNOWLEDGEMENTS}

This work was supported by grants from National Natural Science Foundation of China (grant no. 31770273). The authors thank Dr. Xiaochun Ge (Fudan University, Shanghai, China) for her assistance with microscopic observation of DTX6-GFP localization and helpful discussions.

\section{REFERENCE}

Abe, A., Kosugi, S., Yoshida, K., Natsume, S., Takagi, H., Kanzaki, H., Matsumura, H., 
Xia et al.

357

358

359

360

361

362

363

364

365

366

367

368

369

370

371

372

373

374

375

376

377

378

Yoshida, K., Mitsuoka, C., Tamiru, M., et al. (2012). Genome sequencing reveals

agronomically important loci in rice using MutMap. Nat Biotechnol 30:174-178.

Bent., A.F. (2000). Arabidopsis in planta transformation. Uses, mechanisms, and prospects for transformation of other species. Plant Physiol 124:1540-1547.

Chaiwanon, J., Garcia, V.J., Cartwright, H., Sun, Y., and Wang, Z.Y. (2016). Immunophilin-like FKBP42/TWISTED DWARF1 Interacts with the Receptor Kinase BRI1 to Regulate Brassinosteroid Signaling in Arabidopsis. Mol Plant 9:593-600.

Chen, R., Sun, S., Wang, C., Li, Y., Liang, Y., An, F., Li, C., Dong, H., Yang, X., Zhang, J., et al. (2009). The Arabidopsis PARAQUAT RESISTANT2 gene encodes an S-nitrosoglutathione reductase that is a key regulator of cell death. Cell Res 19:1377-1387.

Diener., A.C., Gaxiola., R.A., and Fink., G.R. (2001). Arabidopsis ALF5, a Multidrug Efflux Transporter Gene Family Member, Confers Resistance to Toxins. The Plant Cell 13:1625-1637.

Dobritzsch, M., Lubken, T., Eschen-Lippold, L., Gorzolka, K., Blum, E., Matern, A., Marillonnet, S., Bottcher, C., Drager, B., and Rosahl, S. (2016). MATE Transporter-Dependent Export of Hydroxycinnamic Acid Amides. Plant Cell 28:583-596.

Doshi, R., McGrath, A.P., Pineros, M., Szewczyk, P., Garza, D.M., Kochian, L.V., and Chang, G. (2017). Functional characterization and discovery of modulators of SbMATE, the agronomically important aluminium tolerance transporter from Sorghum bicolor. Sci Rep 7:17996.

Durrett, T.P., Gassmann, W., and Rogers, E.E. (2007). The FRD3-mediated efflux of citrate 

144:197-205.

Fujita, M., Fujita, Y., luchi, S., Yamada, K., Kobayashi, Y., Urano, K., Kobayashi, M., Yamaguchi-Shinozaki, K., and Shinozaki, K. (2012). Natural variation in a polyamine transporter determines paraquat tolerance in Arabidopsis. Proc Natl Acad Sci U S A 109:6343-6347.

Fujita, M., and Shinozaki, K. (2014). Identification of polyamine transporters in plants: paraquat transport provides crucial clues. Plant Cell Physiol 55:855-861.

Green, L.S., and Rogers, E.E. (2004). FRD3 controls iron localization in Arabidopsis. Plant Physiol 136:2523-2531.

Hawkes, T.R. (2014). Mechanisms of resistance to paraquat in plants. Pest Manag Sci $70: 1316-1323$.

Itoh, K., and Miyahara, M. (1984). Inheritance of Paraquat Resistance in Erigeron philadelphicus L. Weed Research 29:301-307.

Kuroda, T., and Tsuchiya, T. (2009). Multidrug efflux transporters in the MATE family. Biochim Biophys Acta 1794:763-768. J.L., Ma, J.F., et al. (2018). Transcription factor WRKY22 promotes aluminum tolerance via activation of OsFRDL4 expression and enhancement of citrate secretion in rice (Oryza sativa). New Phytol 219:149-162. 
intracellular transport of paraquat. Plant Physiol 162:470-483. characterization of a plant efflux carrier for multidrug and heavy metal detoxification. J Biol Chem 277:5360-5368.

Li, N., Meng, H., Xing, H., Liang, L., Zhao, X., and Luo, K. (2017). Genome-wide analysis of MATE transporters and molecular characterization of aluminum resistance in Populus. J Exp Bot 68:5669-5683.

Lu, M. (2016). Structures of multidrug and toxic compound extrusion transporters and their mechanistic implications. Channels (Austin) 10:88-100.

Lu, P., Magwanga, R.O., Guo, X., Kirungu, J.N., Lu, H., Cai, X., Zhou, Z., Wei, Y., Wang, X., Zhang, Z., et al. (2018). Genome-Wide Analysis of Multidrug and Toxic Compound Extrusion (MATE) Family in Gossypium raimondii and Gossypium arboreum and Its Expression Analysis Under Salt, Cadmium, and Drought Stress. G3 (Bethesda) 8:2483-2500.

Ma, Q., Yi, R., Li, L., Liang, Z., Zeng, T., Zhang, Y., Huang, H., Zhang, X., Yin, X., Cai, Z., et al. (2018). GsMATE encoding a multidrug and toxic compound extrusion transporter enhances aluminum tolerance in Arabidopsis thaliana. BMC Plant Biol 18:212. 

and toxic compound extrusion (MATE) family confers aluminum tolerance in sorghum. Nat Genet 39:1156-1161.

Marinova, K., Pourcel, L., Weder, B., Schwarz, M., Barron, D., Routaboul, J.M., Debeaujon, I., and Klein, M. (2007). The Arabidopsis MATE transporter TT12 acts as a vacuolar Plant Cell 19:2023-2038.

Miyauchi, H., Moriyama, S., Kusakizako, T., Kumazaki, K., Nakane, T., Yamashita, K., Hirata, K., Dohmae, N., Nishizawa, T., Ito, K., et al. (2017). Structural basis for xenobiotic extrusion by eukaryotic MATE transporter. Nat Commun 8:1633.

Murgia, I., Tarantino, D., Vannini, C., Bracale, M., Carravieri, S., and Soave, C. (2004). oxide-induced cell death. Plant J 38:940-953. Trends Pharmacol Sci 27:587-593.

Rogers, E.E., and Guerinot, M.L. (2002). FRD3, a member of the multidrug and toxin efflux Arabidopsis thaliana plants overexpressing thylakoidal ascorbate peroxidase show increased resistance to Paraquat-induced photooxidative stress and to nitric family, controls iron deficiency responses in Arabidopsis. Plant Cell 14:1787-1799. citrate effluxer promotes iron nutrition between symplastically disconnected tissues throughout Arabidopsis development. Plant Cell 23:2725-2737. 
Serrano, M., Wang, B., Aryal, B., Garcion, C., Abou-Mansour, E., Heck, S., Geisler, M., Mauch, requires the multidrug and toxin extrusion-like transporter EDS5. Plant Physiol 162:1815-1821.

Suntres., Z.E. (2002). Role of antioxidants in paraquat toxicity. Toxicology 180:65-77.

Szigeti, Z., and Lehoczki, E. (2003). A review of physiological and biochemical aspects of resistance to atrazine and paraquat in Hungarian weeds. Pest Manag Sci 59:451-458.

Takagi, H., Tamiru, M., Abe, A., Yoshida, K., Uemura, A., Yaegashi, H., Obara, T., Oikawa, K., Utsushi, H., Kanzaki, E., et al. (2015). MutMap accelerates breeding of a salt-tolerant rice cultivar. Nat Biotechnol 33:445-449.

Tanaka, Y., Hipolito, C.J., Maturana, A.D., Ito, K., Kuroda, T., Higuchi, T., Katoh, T., Kato, H.E., Hattori, M., Kumazaki, K., et al. (2013). Structural basis for the drug extrusion mechanism by a MATE multidrug transporter. Nature 496:247-251.

Xi, J., Xu, P., and Xiang, C.B. (2012). Loss of AtPDR11, a plasma membrane-localized ABC transporter, confers paraquat tolerance in Arabidopsis thaliana. Plant J 69:782-791.

Yokosho, K., Yamaji, N., and Ma, J.F. (2011). An Al-inducible MATE gene is involved in external detoxification of Al in rice. Plant J 68:1061-1069. transporter required for efficient translocation of iron in rice. Plant Physiol 149:297-305. system for transient gene expression analysis. Nat Protoc 2:1565-1572. 
Xia et al.

467

468

469

470

471

472

473

474

475

476

477

478

479

\section{Supplemental information}

481

482

483

484

485

486

487

488 Bacteriology 182:6694-6697. MutMap analysis.

Figure S2. Identification of $d t x 6$ mutants. and 4-weeks-old plants, respectively.

Yuji Morita, Atsuko Kataoka, Sumiko Shiota, Tohru Mizushima, and Tsuchiya., T. (2000).

NorM of Vibrio parahaemolyticus Is an Na+Driven Multidrug Efflux Pump. Journal Of

Zhang, H., Zhao, F.G., Tang, R.J., Yu, Y., Song, J., Wang, Y., Li, L., and Luan, S. (2017). Two tonoplast MATE proteins function as turgor-regulating chloride channels in Arabidopsis. Proc Natl Acad Sci U S A 114:E2036-E2045.

Zhang, H., Zhu, H., Pan, Y., Yu, Y., Luan, S., and Li, L. (2014). A DTX/MATE-type transporter facilitates abscisic acid efflux and modulates ABA sensitivity and drought tolerance in Arabidopsis. Mol Plant 7:1522-1532.

Zhao, P.X., Miao, Z.Q., Zhang, J., Chen, S.Y., Liu, Q.Q., and Xiang, C.B. (2020). Arabidopsis MADS-box factor AGL16 negatively regulates drought resistance via stomatal density and stomatal movement. J Exp Bot 71:6092-6106.

Figure S1. The mapping of the mutation responsible for pqt15-D phenotype based on

Figure 3S. Relative expression level of DTX6/DTX6-D gene in leaves of 1-week-old

Table S1. Alleles of 2 major paraquat resistance loci in Arabidopsis

Table S2. Primers sequences used in this study. 
FIGURE LEGENDS

490

Figure 1. pqt15-D mutant and mutation identification.

a. Paraquat resistance phenotype of pqt15-D mutant. Wild type (Col-0) and pqt15-D seeds were germinated and grown on MS medium containing $2 \mu \mathrm{M}$ paraquat for 2 weeks before the image was recorded.

b. Genetic analysis of the pqt15-D mutant. Segregation of paraquat resistance in BC1F2 population. Wild type (Col-0) was used as a negative control and pqt15-D as a positive control.

c. Gene structure of the AtDTX6. White and gray boxes represent untranslated regions (UTRs) and exons, respectively, while black lines denote introns.

d. Identification of the mutation in AT2G04100/AtDTX6 of pqt15-D by sequencing. The red box indicates the codon with G-to-A point mutation. All the other 7 mutants (pqt2-2, pqt8-4, pqt28-1, pqt63-1, pqt66-1, pqt72-1 and pqt73-1) have the same mutation in the same gene (Table S1).

Figure 2. Expression pattern of DTX6 and subcellular localization of DTX6.

a. qRT-PCR analysis of DTX6 expression levels in different tissues (root, stem, rosette leaves, cauline leaf, flower, silique, and seed). Values are mean \pm SD $(\mathrm{n}=3)$.

b. Subcellular localization of DTX6 in the root cells in the elongation zone of DTX6pro:DTX6-GFP transgenic plants. Bar $=50 \mu \mathrm{m}$.

c. Subcellular localization of DTX6 in the protoplasts prepared from the roots of DTX6pro:DTX6-GFP transgenic plants.

\section{Figure 3. Enhanced DTX6 confers strong paraquat resistance.}

a. The phenotype of wild type (Col-0), dtx6-1, dtx6-2, OE-9, OE-13, OE-17, and pqt15-D germinated on MS medium containing different paraquat (PQ) concentration $(0,1.0,1.5$, and $2.0 \mu \mathrm{M})$ for 1 week before survival $\%$ and 
518

519

520

521

522

523

524

525

526

527

528

529

530

531

532

533

534

535

536

537

538

539

540

541

542

543

544

545

546

images were recorded.

b. The survival ratio of wild type (Col-0), dtx6-1, dtx6-2, OE-9, OE-13, OE-17, and pqt15-D germinated as in a. Values are mean $\pm \mathrm{SD}(\mathrm{n}=3,25$ seeds per replicate). Different letters indicate significant differences $(\mathrm{P}<0.05$; one-way ANOVA).

c. Paraquat resistance assay. The phenotype of wild type (Col-0), dtx6-1, dtx6-2, OE-9, OE-13, OE-17, and pqt15-D grown in the soil for 4 weeks before (top) and after sprayed with $80 \mu \mathrm{M}$ paraquat (bottom). Images were recorded before and 5 days after paraquat spray.

d. The survival ratio of wild type (Col-0), dtx6-1, dtx6-2, OE-9, OE-13, OE-17 and pqt15- $D$ for the paraquat resistance assay in c. Values are mean $\pm \mathrm{SD}(\mathrm{n}=$ 3, 60 plants per replicate). Different letters indicate significant differences $(\mathrm{P}<$ 0.05; one-way ANOVA).

e. Heterologous expression of DTX6, DTX6-D in E. coli confers paraquat resistance. E. coli Rosetta strain was transformed with the empty pET28a vector (negative control) or with pET28a containing the DTX6 and DTX6-D cDNA, respectively, and grown on solid medium with 0 or $200 \mu \mathrm{M}$ paraquat for $24 \mathrm{~h}$ before images were recorded.

\section{Figure 4. DTX6 effluxes paraquat out of the cell.}

a. Paraquat retention in the protoplasts. Paraquat efflux assay was conducted as described in Materials and Methods. Paraquat retained in the protoplasts was measured in Col-0 and OE-13 lines. Paraquat retention is defined as percent of the loaded ${ }^{14} \mathrm{C}$-paraquat in the protoplasts. Values are mean $\pm \operatorname{SD}(n=3)$. Different letters indicate significant differences ( $\mathrm{P}<0.05$; one-way ANOVA).

b. Paraquat release into the medium from the protoplasts. Paraquat efflux assay was conducted as described in Materials and Methods. Paraquat released into the buffer was measured in Col-0 and OE-13 lines. Paraquat release is defined as percent of the loaded ${ }^{14} \mathrm{C}$-paraquat in the protoplasts. Values are mean $\pm \mathrm{SD}$ 
547

548

549

550

551

552

553

554

555

556

557

558

559

560

561

562

563

564

565

566

567

568

569

570

571

572

573

574

575

$(\mathrm{n}=3)$. Different letters indicate significant differences $(\mathrm{P}<0.05$; one-way ANOVA).

c. E. coli cells expressing DTX6 and DTX6-D release more paraquat into medium than control (pET28a empty vector). Paraquat release is defined as percent of the loaded ${ }^{14} \mathrm{C}$-paraquat in bacterial cells. Values are mean $\pm \operatorname{SD}(n=3)$. Different letters indicate significant differences ( $\mathrm{P}<0.05$; one-way ANOVA).

Figure 5. Increased negative charge on the surface of the substrate entry domain may promotes paraquat transport of DTX6-D

a. The structure model of DTX6 simulated by SWISS-MODEL from structure of AtDTX14 (Side view). The transmembrane helices are labeled and colored in rainbow. The residue Gly311 was indicated by black sphere. The red horizontal lines denote membrane with extracellular face (Out) and intracellular face (In).

b. Top view of DTX6.

c. Electrostatic surface potential map of DTX6. Gly311 is located in the entrance of transporter tunnel, the hydrophobic patch of Gly311 is highlighted by the black circle. The scale ranges from $-50 \mathrm{kT} / \mathrm{e}$ (red) to $50 \mathrm{kT} / \mathrm{e}$ (blue).

d. Electrostatic surface potential map of DTX6-D. Glu311 is located in the entrance of transporter tunnel, the negative charge patch of G311E is highlighted by red circle. The scale ranges from $-50 \mathrm{kT} / \mathrm{e}$ (red) to $50 \mathrm{kT} / \mathrm{e}$ (blue).

e. The $311^{\text {th }}$ amino acid of DTX6 protein was mutated to Glu, Asp and Gln, respectively.

f. E. coli Rosetta cells were transformed with the empty pET28a vector (negative control), or with pET28a containing the DTX6 wild type cDNA or DTX6-D, DTX6-Asp, and DTX6-Gln cDNA with the mutation causing G311E, G311D, and G311N amino acid change respectively, and grown on solid medium with 0 and $200 \mu \mathrm{M}$ paraquat for $24 \mathrm{~h}$ before images were recorded. 
bioRxiv preprint doi: https://doi.org/10.1101/2021.02.07.430154; this version posted February 8, 2021. The copyright holder for this preprint (which was not certified by peer review) is the author/funder. All rights reserved. No reuse allowed without permission.

Xia et al.

576 
$\mathbf{a}$

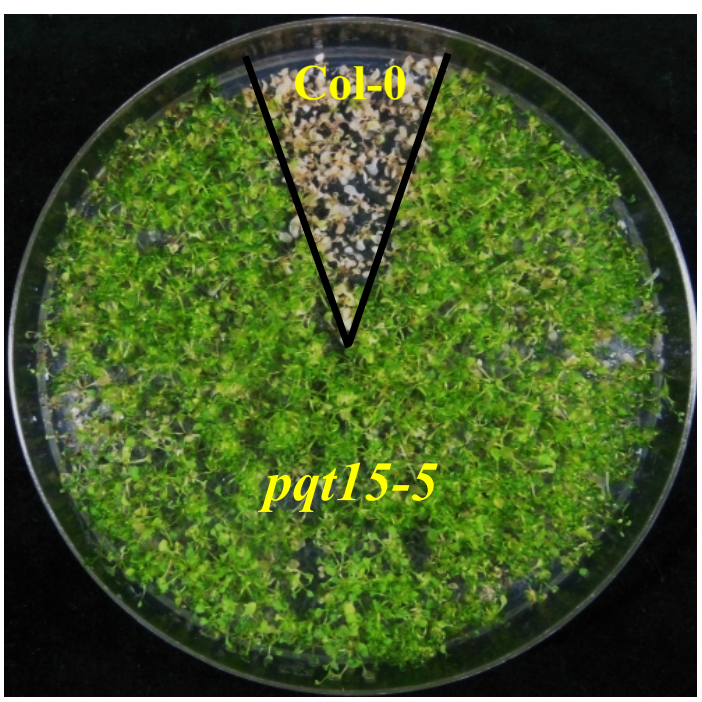

b

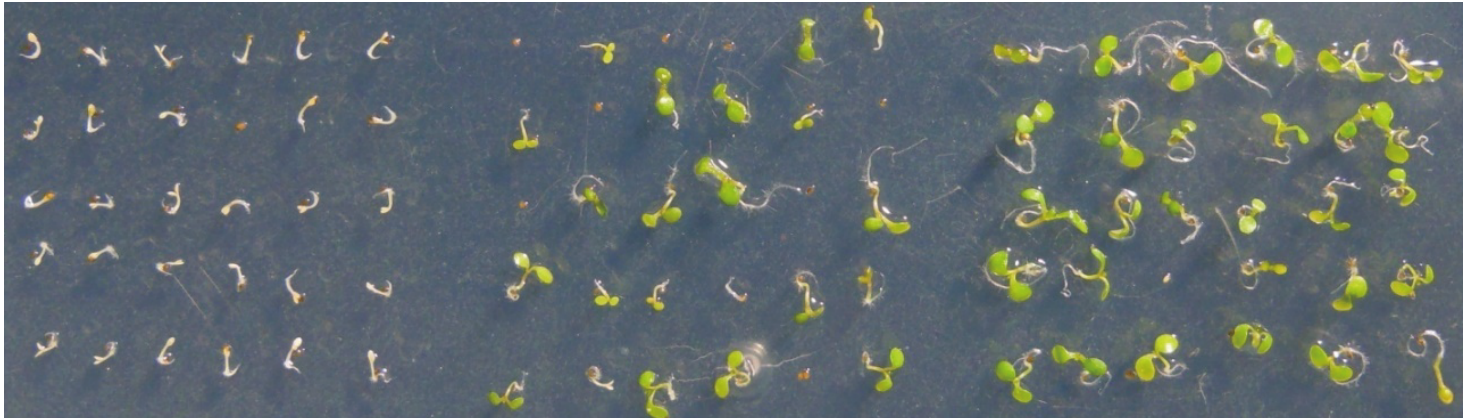

Col-0

5'

d
C

AT2G04100 (AtDTX6)
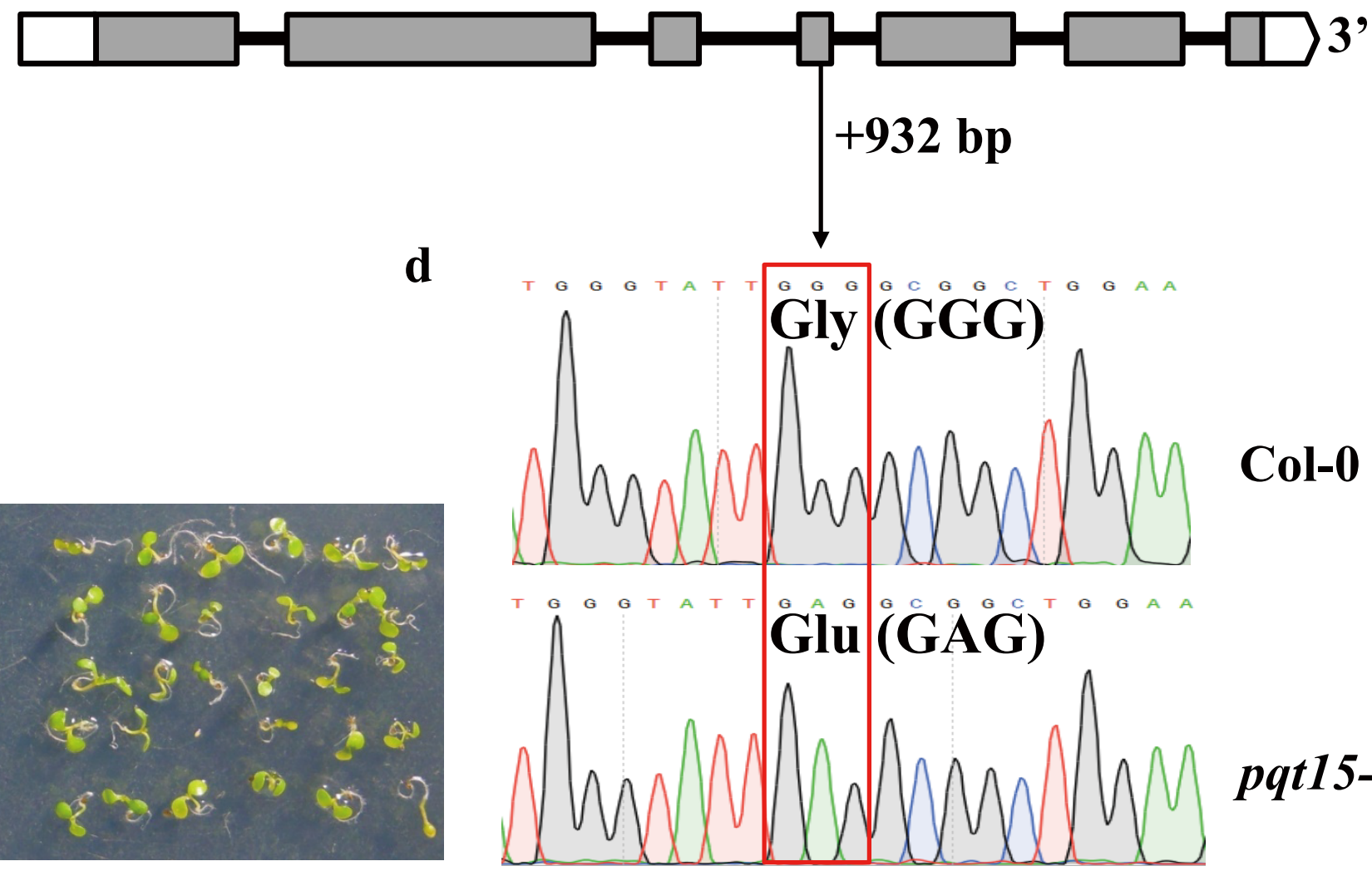

Col-0

pqt15-D

Figure 1.pqt15-D mutant and mutation identification.

a. $\quad$ Paraquat resistance phenotype of pqt15-D mutant. Wild type (Col-0) and pqt15-D seeds were germinated and grown on MS medium containing $2 \mu \mathrm{M}$ paraquat for 2 weeks before the image was recorded.

b. Genetic analysis of the pqt15-D mutant. Segregation of paraquat resistance in BC1F2 population. Wild type (Col-0) was used as a negative control and pqt15-D as a positive control.

c. Gene structure of the AtDTX6. White and gray boxes represent untranslated regions (UTRs) and exons, respectively, while black lines denote introns.

d. Identification of the mutation in AT2G04100/AtDTX6 of pqt15-D by sequencing. A red box indicates the codon with G-to-A point mutation. All the other 7 mutants (pqt2-2, pqt8-4, pqt28-1, pqt63-1, pqt66-1, pqt72-1 and pqt73-1) have the same mutation in the same gene (Table S1). 
DIC
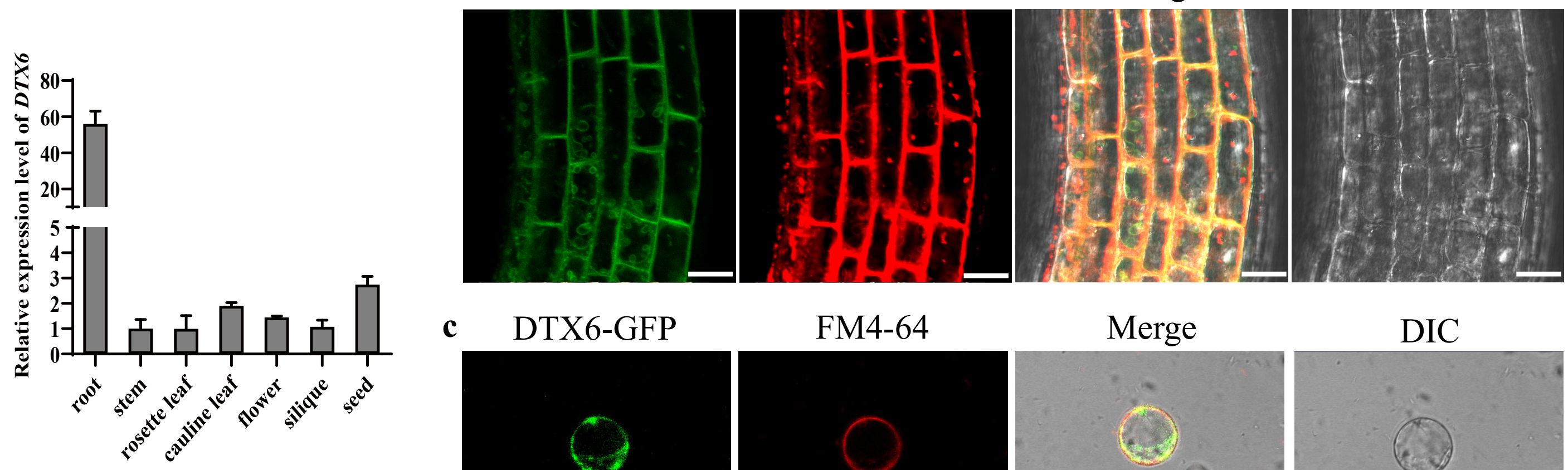
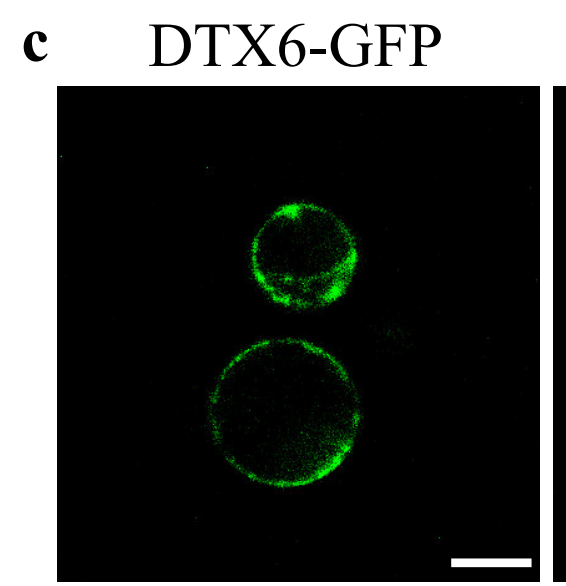

FM4-64

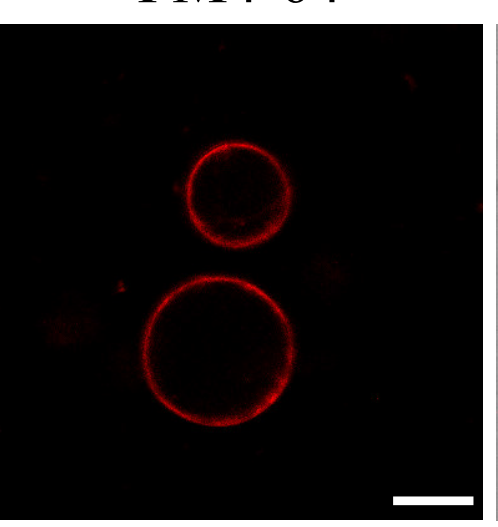

DIC

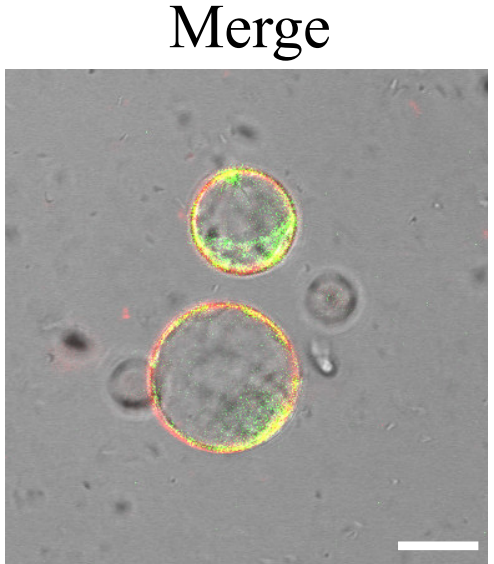

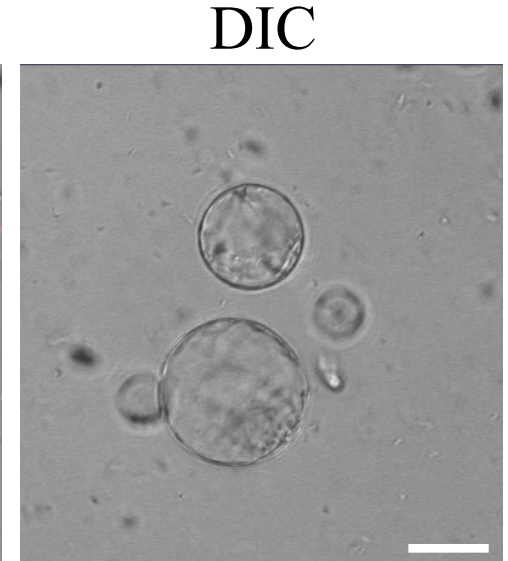

Figure 2. Expression pattern of DTX6 and subcellular localization of DTX6.

a. $\quad$ qRT-PCR analysis of DTX6 expression levels in different tissues (root, stem, rosette leaves, cauline leaf, flower, silique, and seed). Values are mean $\pm \mathrm{SD}(\mathrm{n}=3)$.

b. Subcellular localization of DTX6 in the root cells in the elongation zone of DTX6pro:DTX6-GFP transgenic plants. Bar $=50 \mu \mathrm{m}$.

c. Subcellular localization of DTX6 in the protoplasts prepared from the roots of DTX6pro:DTX6-GFP transgenic plants. 


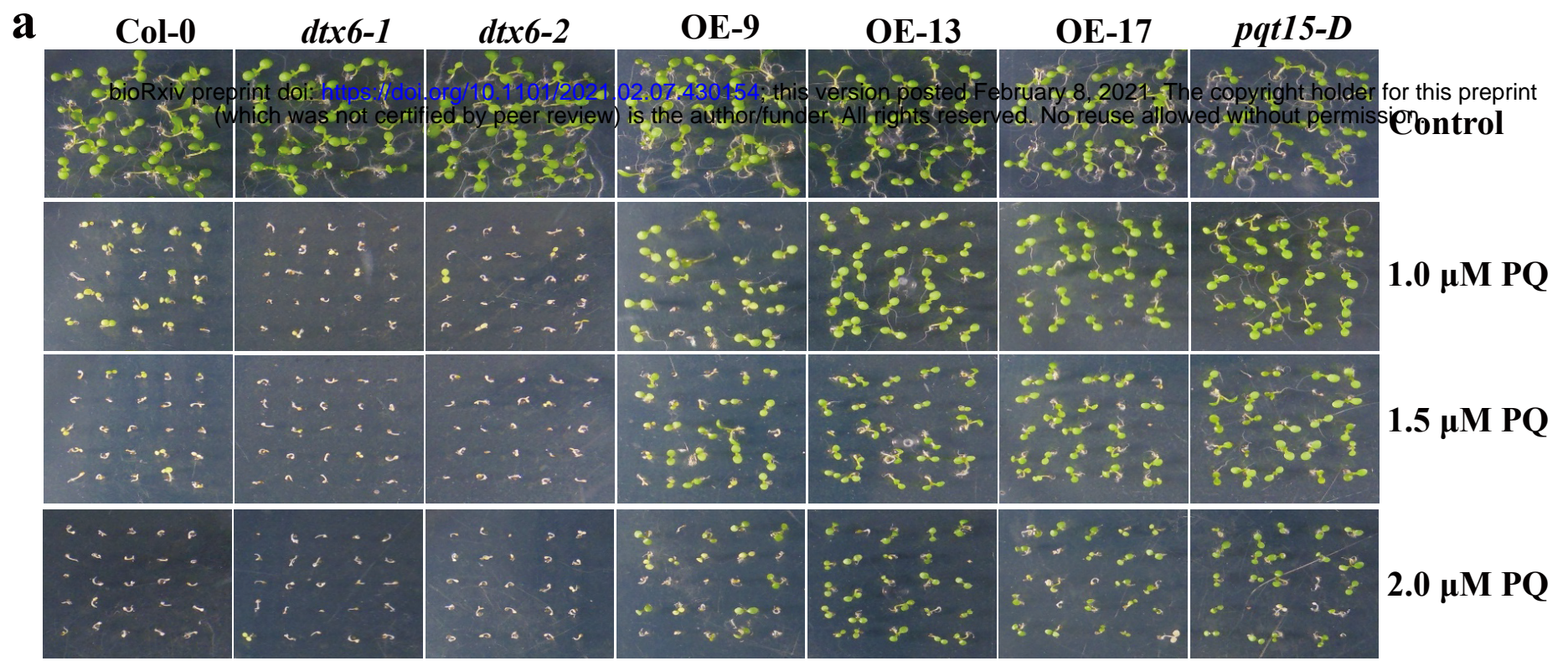

c

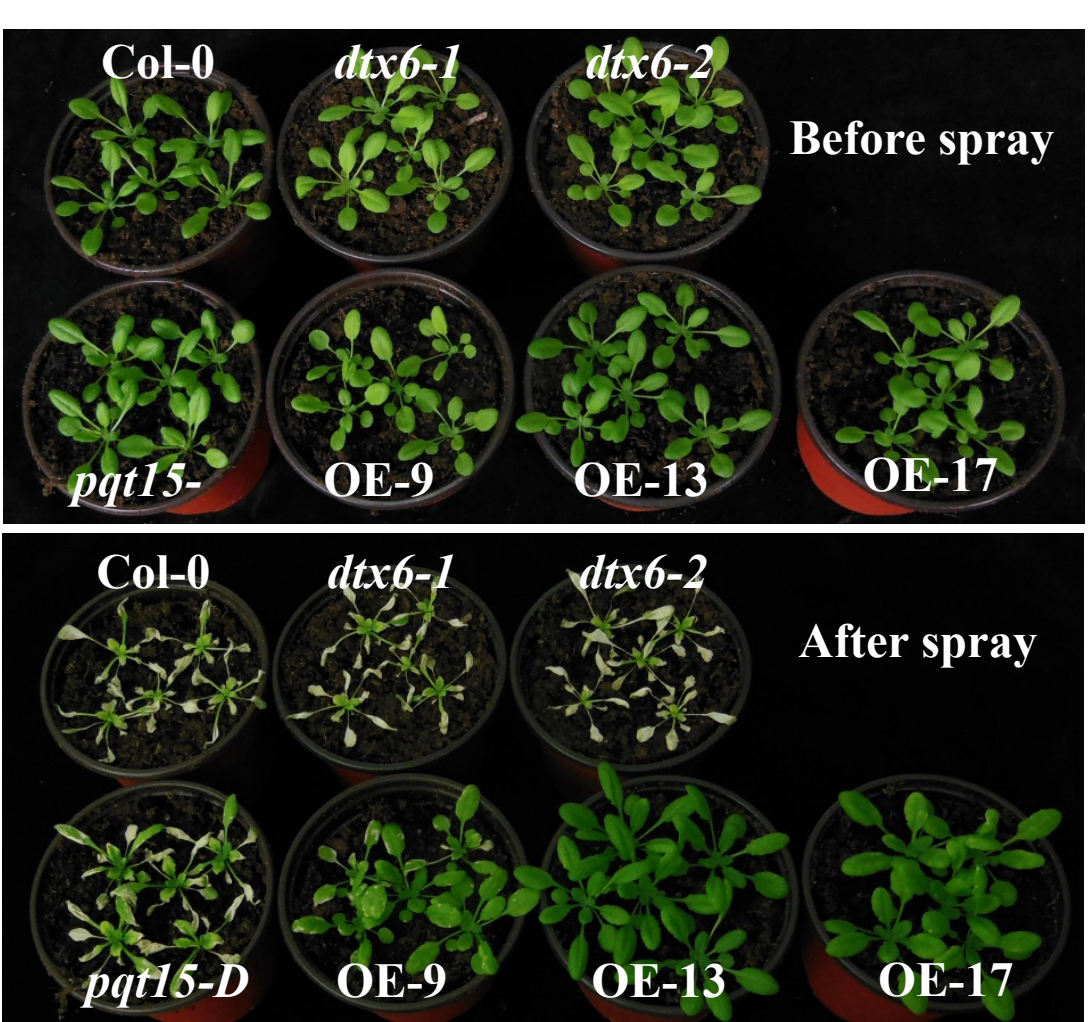

\section{b}

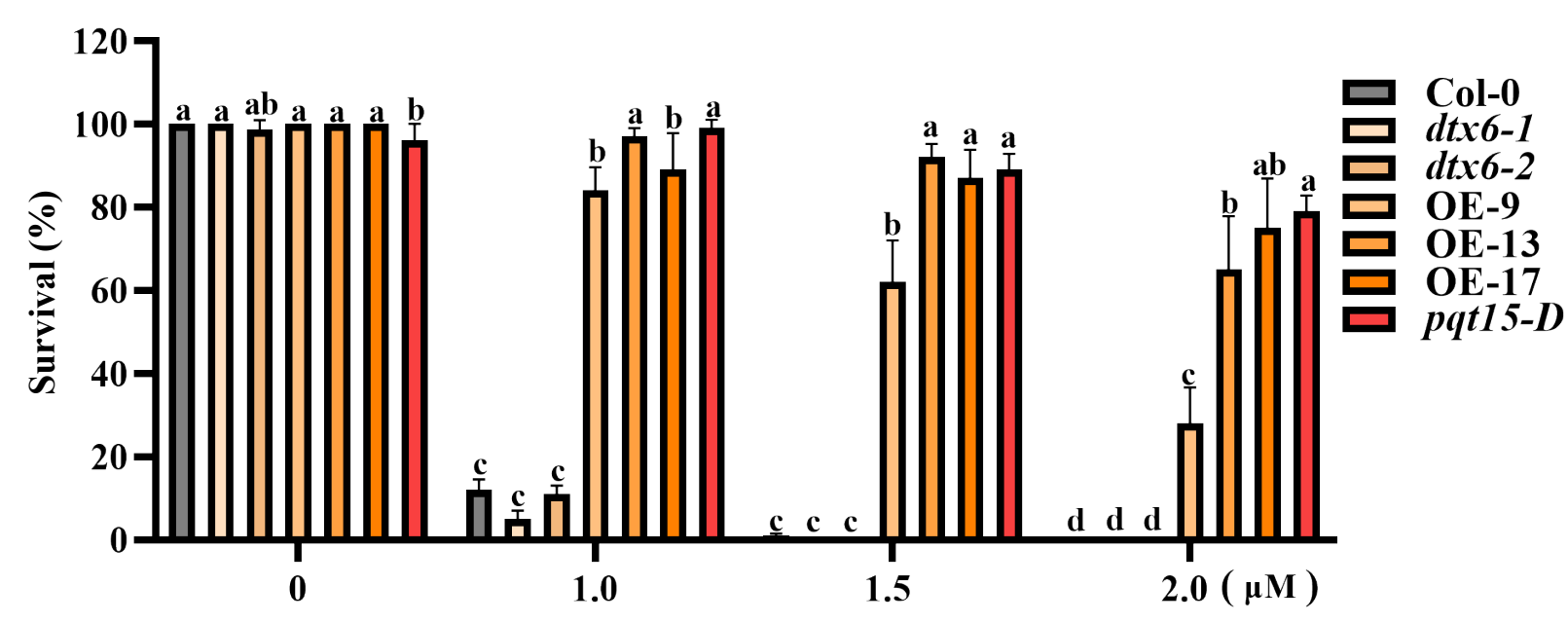

d

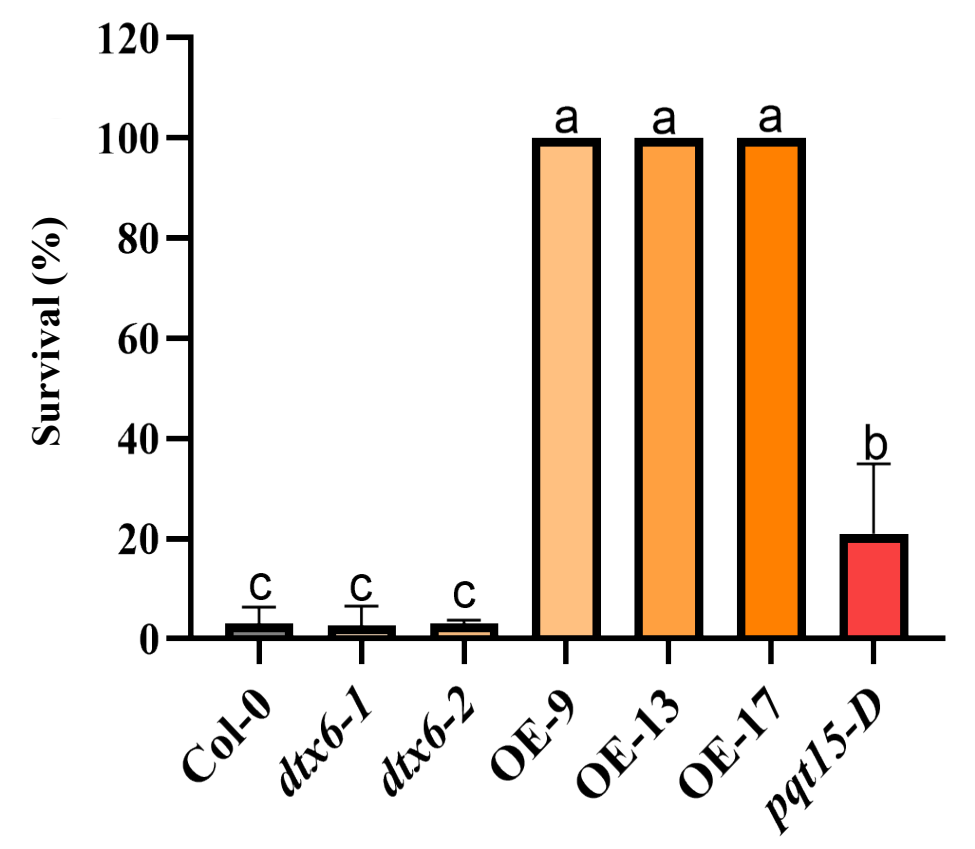

e

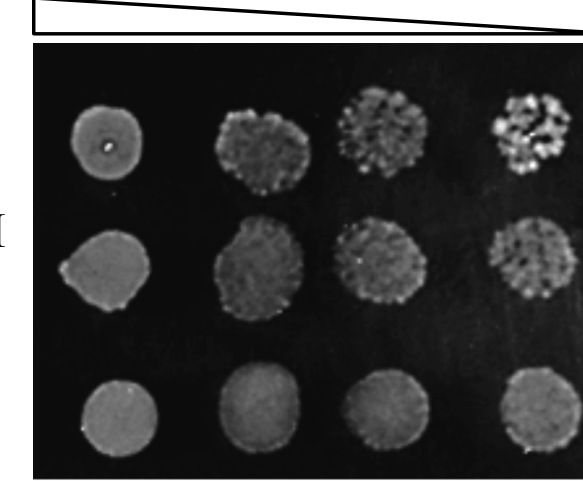

pET28a

pET28a:DTX6

pET28a:DTX6-D

$200 \mu M$

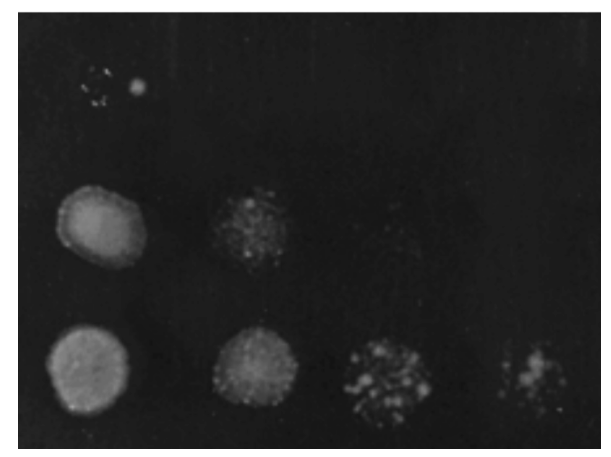

pET28a

pET28a:DTX6

pET28a:DTX6-D

Figure 3. Enhanced DTX6 confers strong paraquat resistance.

a. The phenotype of wild type (Col-0), $d t x 6-1, d t x 6-2$, OE-9, OE-13, OE-17, and pqt15-D germinated on MS medium containing different paraquat (PQ) concentration $(0,1.0,1.5$, and $2.0 \mu \mathrm{M})$ for 1 week.

b. The survival ratio of wild type (Col-0), $d t x 6-1, d t x 6-2$, OE-9, OE-13, OE-17, and pqt15-D. Values are mean $\pm \mathrm{SD}(\mathrm{n}=3,25$ seeds per replicate). Different letters indicate significant differences $(\mathrm{P}<0.05$; one-way ANOVA).

c. Paraquat resistance assay. The phenotype of wild type (Col-0), $d t x 6-1, d t x 6-2$, OE-9, OE-13, OE-17 and pqt15-D grown in the soil for 4 weeks (top) and sprayed with $80 \mu \mathrm{M}$ paraquat (bottom). Images were recorded 5 days after paraquat spray.

d. The survival ratio of wild type (Col-0), $d t x 6-1, d t x 6-2$, OE-9, OE-13, OE-17 and pqt15-D. Values are mean $\pm \mathrm{SD}$ ( $\mathrm{n}=3,60$ plants per replicate). Different letters indicate significant differences $(\mathrm{P}<0.05$; one-way ANOVA).

e. Heterologous expression of DTX6, DTX6-D in E. coli confers paraquat resistance. E. coli Rosetta cells were transformed with the empty pET28a vector (negative control) or with pET28a containing the DTX6 and DTX6-D cDNA, respectively, and grown on solid medium with 0 and $200 \mu \mathrm{M}$ paraquat for $24 \mathrm{~h}$ before images were recorded. 


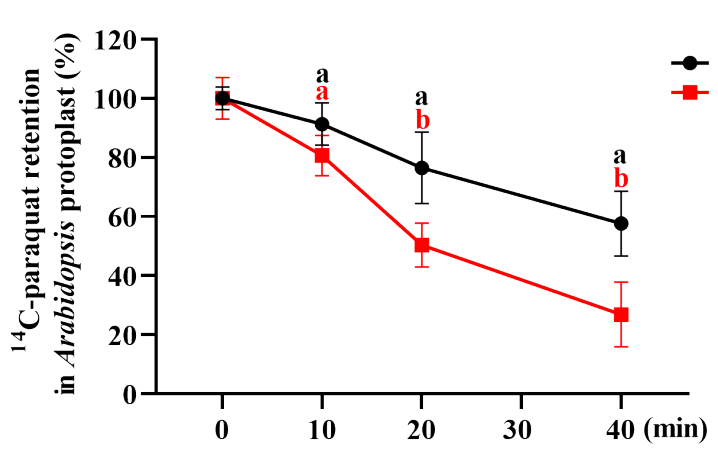

b

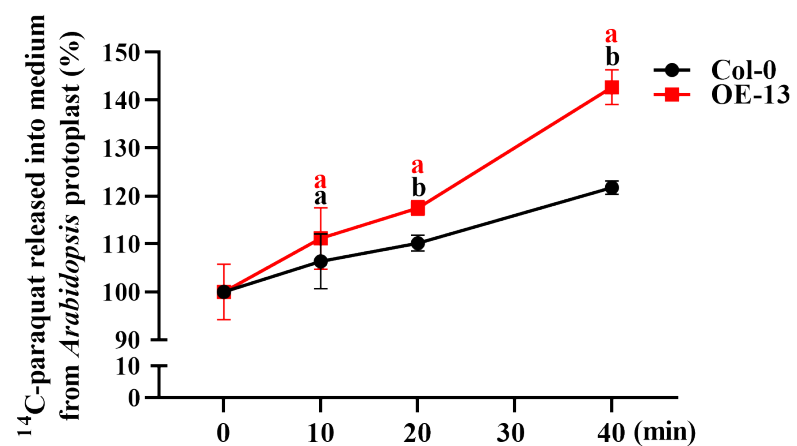

c

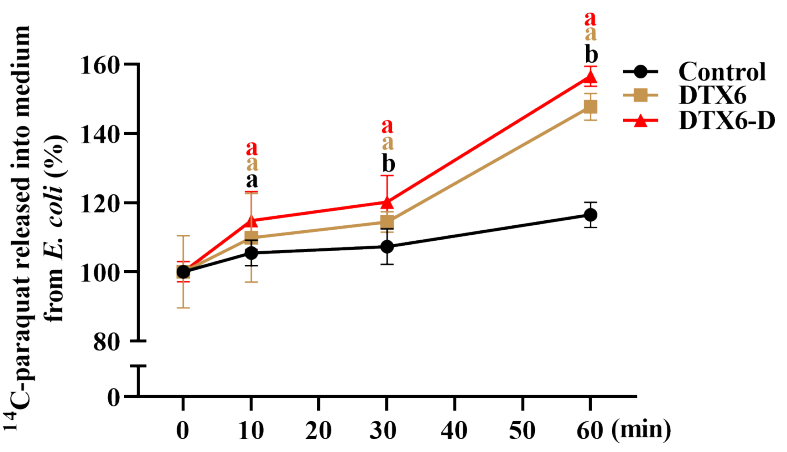

Figure 4. DTX6 effluxes paraquat out of the cell.

a. Paraquat retention in the protoplasts. Paraquat efflux assay was conducted as described in Materials and Methods. Paraquat retained in the protoplasts was measured in Col-0 and OE-13 lines. Paraquat retention is defined as percent of the loaded ${ }^{14} \mathrm{C}$-paraquat in the protoplasts. Values are mean $\pm \mathrm{SD}(\mathrm{n}=3)$. Different letters indicate significant differences $(\mathrm{P}<$ 0.05 ; one-way ANOVA).

b. Paraquat release into the medium from the protoplasts. Paraquat efflux assay was conducted as described in Materials and Methods. Paraquat released into the buffer was measured in Col-0 and OE-13 lines. Paraquat release is defined as percent of the loaded ${ }^{14} \mathrm{C}$-paraquat in the protoplasts. Values are mean $\pm \mathrm{SD}(\mathrm{n}=3)$. Different letters indicate significant differences $(\mathrm{P}<0.05$; one-way ANOVA).

c. $\quad$ E. coli cells expressing DTX6 and DTX6-D release more paraquat into medium than control (pET28a empty vector). Paraquat release is defined as percent of the loaded ${ }^{14} \mathrm{C}$-paraquat in bacterial cells. Values are mean $\pm \mathrm{SD}(\mathrm{n}=3)$. Different 


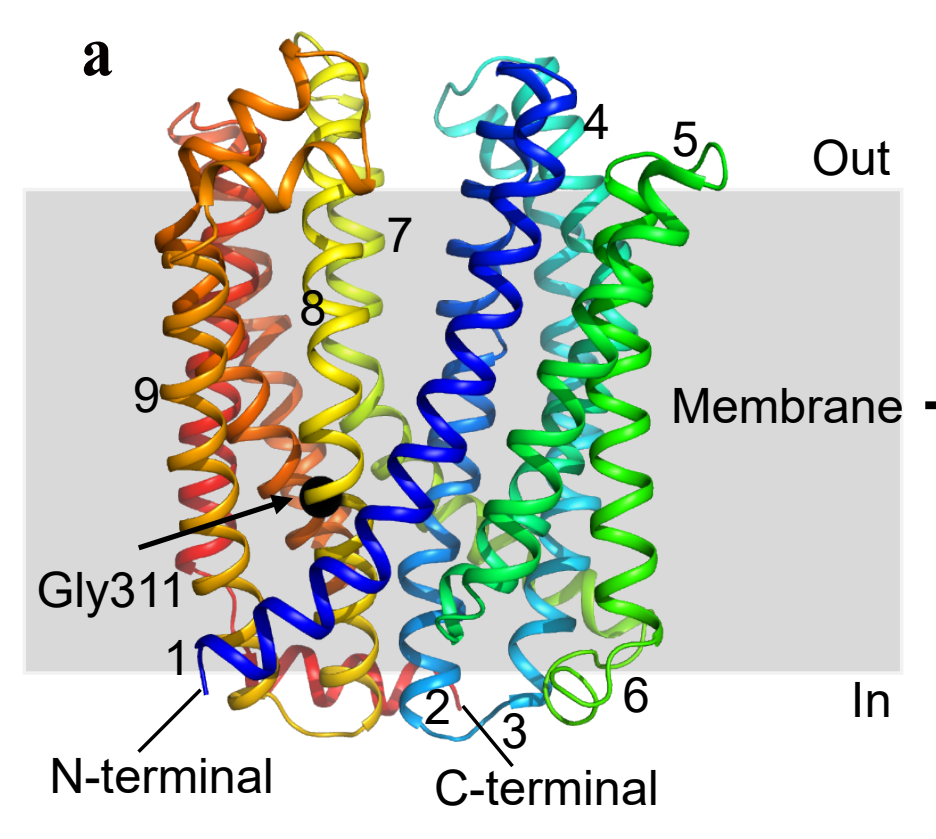

e

\begin{tabular}{|l|l|l|}
\hline & 311 $^{\text {th }}$ Amino acid & side chain \\
\hline DTX6 & Glycine (Gly) & $-\mathrm{H}$ \\
\hline DTX6-D & Glutamic acid (Glu) & $-\left(\mathrm{CH}_{2}\right)_{2}-\mathrm{COOH}$ \\
\hline DTX6-Asp & Aspartic acid (Asp) & $-\mathrm{CH}_{2}-\mathrm{COOH}$ \\
\hline DTX6-Gln & Glutamine (Gln) & $-\left(\mathrm{CH}_{2}\right)_{2}-\mathrm{CO}-\mathrm{NH}_{2}$ \\
\hline
\end{tabular}

b
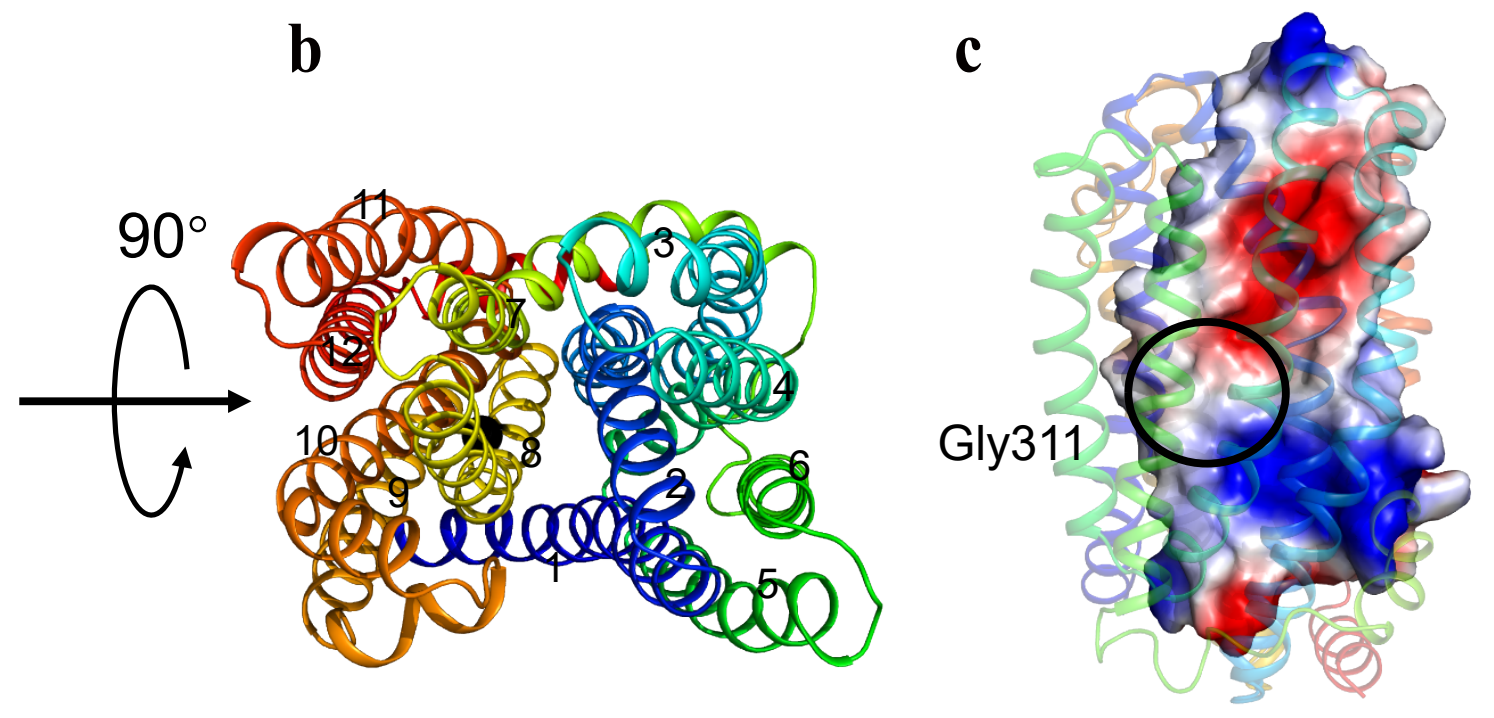

f

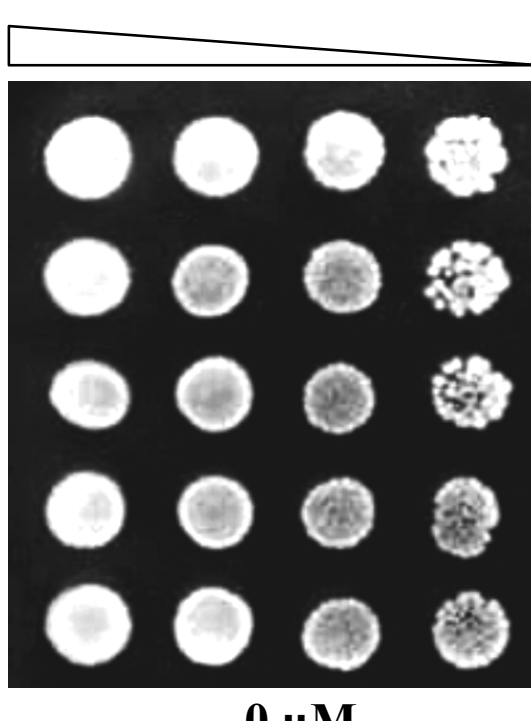

Gly311

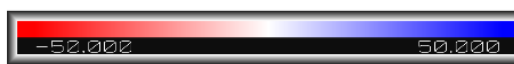

d G311E

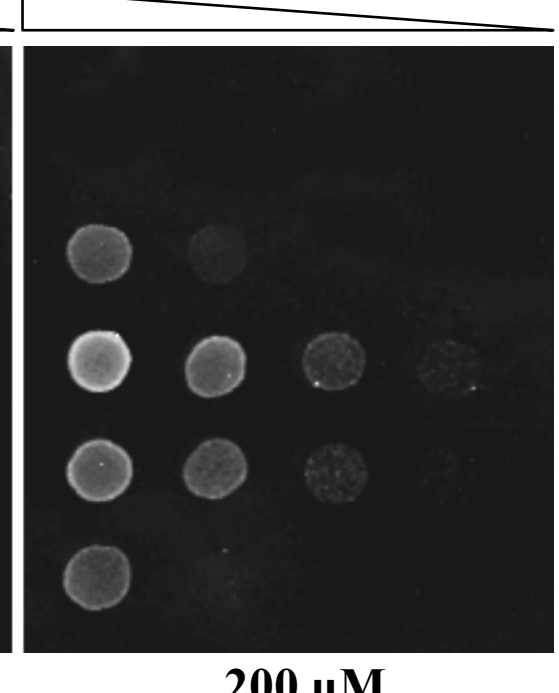

pET28a

pET28a:DTX6

pET28a:DTX6-D

pET28a:DTX6-Asp

pET28a:DTX6-GIn

Figure 5. Increased negative charge on the surface of the substrate entry domain may promotes paraquat transport of DTX6-D

a. The structure model of DTX6 simulated by SWISS-MODEL from structure of AtDTX14 (Side view). The transmembrane helices are labeled and colored in rainbow. The residue Gly311 was indicated by black sphere. The red horizontal lines denote membrane with extracellular face (Out) and intracellular face (In).

\section{b. Top view of DTX6.}

c. Electrostatic surface potential map of DTX6. Gly311 is located in the entrance of transporter tunnel, the hydrophobic patch of Gly311 is highlighted by the black circle. The scale ranges from $-50 \mathrm{kT} / \mathrm{e}$ (red) to $50 \mathrm{kT} / \mathrm{e}$ (blue).

d. Electrostatic surface potential map of DTX6-D. Glu311 is located in the entrance of transporter tunnel, the negative charge patch of G311E is highlighted by red circle. The scale ranges from $-50 \mathrm{kT} / \mathrm{e}$ (red) to $50 \mathrm{kT} / \mathrm{e}$ (blue).

e. $\quad$ The 311th amino acid of DTX6 protein was mutated to Glu, Asp and Gln, respectively.

f. $\quad$ E. coli Rosetta cells were transformed with the empty pET28a vector (negative control), or with pET28a containing the DTX6 wild type cDNA or DTX6-D, DTX6-Asp, and DTX6-Gln cDNA with the mutation causing G311E, G311D, and G311N amino acid change respectively, and grown on solid medium with 0 and $200 \mu \mathrm{M}$ paraquat for $24 \mathrm{~h}$ before images were recorded. 